\title{
Definition of Post-COVID-19 General practice / family medicine
}

Filipe Prazeres

Potential competing interests: The author(s) declared that no potential competing interests exist.

General practice / family medicine is defined as the medical speciality that is attentive to the overall health and well-being of patients, from birth to death, and manages common acute and chronic health conditions of patients of all ages, either in-person or by telemedicine based on patients' preferences and medical needs. 